\title{
Principle of Spectral Reciprocity in Biomechanics of Locomotion and Rehabilitation
}

\author{
Mark Pitkin*
}

Department of Physical Medicine and Rehabilitation, Tufts University School of Medicine, Boston, MA 02111, USA

\begin{abstract}
Providing the technical means to prevent collapse or falls in patients with different types of pathology in motion and balance control is one of the traditional problems in rehabilitation engineering. A means of addressing the problem using assistive devices, including prostheses, is to restrict the mobility in certain anatomical or artificial joints by applying corsets, braces, brakes and locks. The restriction of mobility in the joints increases local stiffnesses, and in a sense, tunes the spectrum of oscillations in these joints out of resonantly dangerous zones. So far, these efforts for limiting unwanted mobility are mostly empirical within rehabilitation technologies, and we suggest that they can be optimized with algorithms for controlling the spectrum of oscillations used in multi-linked technical systems. Further, we suggest that tuning out of resonance is inseparable from the phenomenon of tuning into resonance that is widely recognized in biomechanics of locomotion. These considerations result in our postulating the principle of spectral reciprocity in locomotion.
\end{abstract}

Key Words: rehabilitation engineering, spectral optimization gait, locomotion, resonance antiresonance.

\section{INTRODUCTION}

The concept of resonance in the biomechanics of locomotion has a long history [1]. Resonance is widely recognized as useful for minimizing the energy cost of propelling the body segments. Knowing how to tune into resonance is essential in acquiring skills and refining training in the martial arts and in sports like skating, tennis and gymnastics. To match the limbs' natural frequencies, the motor control system regulates the moments of inertia of the limbs and changes the stiffness in the joints - thus tuning into resonance. During the swing phase of normal gait the knee, hip and elbow angles change the magnitude depending on the speed of gait. This change in angle allows the leg to articulate with minimal muscle drive, at any given speed of gait $[2$, 3].

Here we characterize gait as a sequence of coordinated restricted and unrestricted mobility in the body's joints (Aristotle was probably the first to propose such an approach [4, 5]). We believe that under this characterization, unrestricted mobility is not more important than restricted mobility. Rather, the opposite is true, considering the permanence of the task of preventing collapsing due to gravity. This brings us to our assumption that there exists a mechanism for simultaneous controlling both the tuning into, and also the tuning out of resonance. Since muscles perform the necessary restriction/fixation of joint articulation within the joint's range of the motion, facilitating the muscles' efforts with properly designed assistive devices would minimize total muscle work.

In prosthetics, there were many attempts to incorporate tuning into resonance into prosthesis's features. Otto Bock manufactures a C-Leg prosthesis, which adjusts its natural

*Address correspondence to this author at the Department of Physical Medicine and Rehabilitation, Tufts University School of Medicine, Boston, MA 02111, USA; E-mail: MPitkin@tufts-nemc.org frequency during the swing phase using load activated feedback [6]. However, resonance is undesirable when a joint's mobility has to be restricted in accordance with the structure of locomotion.

\section{SPECTRAL OPTIMIZATION IN ASSISTIVE DE- VICES}

A prosthetic device is a multi-linker, whose natural frequencies can be calculated during the design process. We suggest that the spectral analysis should be a part of a design in prosthetics and orthotics similar to those of analysis of instability in rotor-shaft mechanisms [7]. Since a limb prosthesis serves as a substitute for several anatomical joints, it can be included in the system of spectral optimization, which as we suggest, functions in the whole body. As a consequence, optimization can be achieved by controlling only a few of the stiffnesses [8], similarly to mechanical systems [9, $10]$.

Consider the biped gait of a poly-linker whose dynamics are described by the system of differential equations

$$
A u^{\prime \prime}+B u^{\prime}=C u=f, \quad C=C(\lambda(t)),
$$

where $A, B, C$ are symmetric matrices of kinetic energy, dispersion and potential energy respectively, and $\lambda(t)$ is a controlled vector function of rigidities in the joints of the polylinker.

We refer to previous works where the stride has been divided based on the dominant mobility in metatarsal, ankle and hip joints [11-13]. In each of these phases, the human body is approximated by a system with one degree of freedom. Each of the allowed configurations has its own natural frequency $\omega^{*}$, which prompts the body to tune into resonance-type mobility in one or a few joints. At the same time, the body must adjust its entire spectrum to assure that the joints, which have to be locked, are not affected by $\omega^{*}$. In other words, $\omega^{*}$ is not only the resonantly advantageous 
frequency, but is also resonantly dangerous with respect to the integrity of the current body's configuration. That consideration reduces the problem of gait control mathematically to the problem of control over the spectrum of an operator pencil corresponding to the system of equations (1). The mathematical problem is solved variationally [8] in terms of abstract Rayleigh systems [10]. The search for a solution $u=x \exp (\omega t),(x \neq 0)$ for the homogeneous equation corresponding to equation (1), results in the equation $\omega^{2} A x+\omega B x+C x=0$, generating the pencil of matrices

$$
L(\omega)=\omega^{2} A+\omega B+C
$$

The problem of control over the spectrum is formulated as follows: for a given Rayleigh system $R_{\lambda}\left\{L_{\lambda}, p_{\lambda}\right\}$ depending on the control parameter $\lambda \in U$ and the set $\Omega \supseteq \omega^{*}$, it is necessary to choose $\lambda \in U$ so that the spectrum $\sigma\left(R_{\lambda}\right)$ does not meet $\Omega$.

In that statement of the problem, the Rayleigh system depends linearly on the controlling parameter, and the spectrum of the pencil (2) breaks down into two sets of values of the Rayleigh functional, according to the condition of strong damping [14]:

$$
p_{ \pm}(x)=\frac{-(B x, x) \pm \sqrt{(B x, x)^{2}-4(A x, x)(C x, x)}}{2(A x, x)}
$$

The natural frequencies of each model's configuration are real positive. Therefore, the solvability criterion for the problem of tuning out of the resonantly dangerous domain $\Omega=[\beta,+\infty)$ has the form: $L(0)>0$ on the set $V=\left\{x_{i}\right\},(x \neq 0)$. After substituting zero in the equation (2), we get finally the criterion of tuning out of resonance as

$$
\left\{\begin{array}{l}
\left(C_{i} x, x\right) \geq 0, \quad \text { where } i-\text { indexes for which } \lambda_{i} \geq 0 \\
\left(C_{i} x, x\right)=0, \quad \text { where } i-\text { indexes for which } \lambda_{i}<0
\end{array}\right.
$$

If $\Omega_{+}=[\beta,+\infty), \Omega_{-}=(-\infty, \beta)$, and $\left(q_{+}(\lambda), q_{\bar{L}}(\lambda)\right)$ is the maximal (minimal) point of the spectrum of $\bar{L}_{\lambda}$, then the distance $\rho$ from $q_{ \pm}$to $\Omega_{ \pm}$is given by the formula

$$
\rho \equiv \sup _{\Lambda} \operatorname{dist}\left\{\sigma\left(R_{\lambda}\right), \Omega_{ \pm}\right\}=\inf _{x \in V}|p(x)-\beta|
$$

where $\Lambda=\{\lambda\}$.

As we consider $\Omega=\Omega_{+}$, the formula (4) is transformed into $\rho=\inf _{x \in V}|p(x)-\beta|$,

and since $\beta=0$, finally

$$
\rho=\min _{V_{+}}\left[\frac{-(B x, x) \pm \sqrt{(B x, x)^{2}-4(A x, x)(C x, x)}}{2(A x, x)}\right]
$$

By substituting the entries of the matrices $A, B$, and $C$ in (4) and (5) we can obtain the unknown values of the components of a piecewise constant controlling vector-function for each phase of the stride. Thus, to avoid the resonantly dangerous domain of the natural frequencies, the stiffnesses in prosthesis' joints have to be designed to meet criteria (4)-(5).

For prosthetic components which don't have distinctive joints but have the shape of a shell, we propose a model of tuning out of dangerous resonance by using a cylindrical closed shell of radius $R$ and length $l$ [15]. We include enforcement ribs in the cylindrical shell, which resemble the high counters in orthopedic shoes or a prosthetic socket, whose geometrical parameters influence natural frequencies of the shell.

Let $u, v, w$ be the displacements in three mutually orthogonal directions of the points along the rib $l$. Let $F$ be the square of the rib's cross-section, and $m$ be the total number of ribs.

Displacements should satisfy the boundary conditions and the LaGrange condition [16]:

$$
\frac{\partial(U-T)}{\partial q^{*}}=0
$$

where $q^{*}$ is the generalized coordinate. Potential energy $U$ in (6) is

$$
\begin{aligned}
& U=\frac{E h}{1\left(1-v^{2}\right)} \int_{0}^{2 \pi} \int_{0}^{1 / R}\left\{\begin{array}{l}
\left(\frac{\partial u}{\partial \alpha}\right)^{2}+\left(\frac{\partial v}{\partial \beta}+w\right)^{2}+2 v \frac{\partial u}{\partial \alpha}\left(\frac{\partial v}{\partial \beta}+w\right)+ \\
+\frac{1-v}{2}\left(\frac{\partial u}{\partial \beta}+\frac{\partial v}{\partial \alpha}\right)^{2}
\end{array}\right\} d \alpha d \beta+ \\
& \frac{D}{2 R^{2}} \int_{0}^{2 \pi} \int_{0}^{i / R}\left\{\begin{array}{l}
\left(\frac{\partial^{2} w}{\partial \alpha^{2}}\right)^{2}+\left(\frac{\partial^{2} w}{\partial \beta^{2}}+w\right)^{2}+2 v \frac{\partial^{2} w}{\partial \alpha^{2}}\left(\frac{\partial^{2} w}{\partial \beta^{2}}+w\right)+ \\
+(1-v)\left[\left(\frac{\partial^{2} w}{\partial \alpha \partial \beta}+\frac{\partial u}{\partial \beta}\right)^{2}+\left(\frac{\partial v}{\partial \alpha}-\frac{\partial^{2} w}{\partial \alpha \partial \beta}\right)-\frac{1}{2}\left(\frac{\partial v}{\partial \alpha}+\frac{\partial u}{\partial \beta}\right)^{2}\right]
\end{array}\right\} d \alpha d \beta+ \\
& \frac{E_{1}}{2 R^{3}} \sum_{1}^{m} \int_{0}^{1 / R}\left\{I_{y}\left(\frac{\partial^{2} w}{\partial \alpha^{2}}\right)^{2}+F R^{2}\left(\frac{\partial u}{\partial \alpha}+k \frac{\partial^{2} w}{\partial \alpha^{2}}\right)^{2}\right\} d \alpha,
\end{aligned}
$$

where $E$ and $E_{1}$ are the modulus of elasticity for the shell and the ribs' material, $v$ - Poisson coefficient, $k$ - distance between the center of mass of the rib's cross-section and its median divided by $R$.

Kinetic energy $T$ of oscillation is:

$$
\begin{aligned}
& T=\frac{h \gamma R^{2}}{2 g} \int_{0}^{2 \pi} \int_{0}^{l / R}\left\{\left(\frac{\partial u}{\partial t}\right)^{2}+\left(\frac{\partial v}{\partial t}\right)^{2}+\left(\frac{\partial w}{\partial t}\right)^{22}\right\} d \alpha d \beta+ \\
& \frac{\gamma_{1} R}{2 g} \sum_{1}^{m} \int_{0}^{1 / R}\left\{{ }_{0}\left[\begin{array}{l}
\left(\frac{\partial u}{\partial t}+k \frac{\partial}{\partial t} \frac{\partial w}{\partial \alpha}\right)^{2}+\left(\frac{\partial v}{\partial t}+k \frac{\partial}{\partial t} \frac{\partial w}{\partial \beta}\right)^{2}+ \\
+\left(\frac{\partial^{2} w}{\partial t^{2}}\right)^{2}+\frac{I_{r}}{R^{2}}\left(\frac{\partial}{\partial t} \frac{\partial w}{\partial \beta}\right)^{2}
\end{array}\right]\right\} d \alpha,
\end{aligned}
$$

where $\gamma, \gamma_{1}$ are densities of the shell and the ribs' material respectively.

Analysis of equations (6)-(8) shows that by varying the dimensions of the shell and the ribs, it is possible either to increase or decrease the values of their natural frequencies. This is explained by the asymmetrical change in potential (7) and kinetic (8) energy, and allows us to meet the criterion (4) of tuning out of dangerous zones of the spectrum. As we discussed earlier, the term "dangerous" refers to the situation when a given joint that must be locked at a certain phase of stride, can be affected by the resonance frequency $\omega^{*}$, which is beneficial for the free mobility of another joint, but which compromises the locking of the first joint. 
Tuning a spectrum of a particular joint out of a resonantly dangerous frequency $\omega^{*}$ using the algorithms presented does not compromise the frequencies that can be resonantly useful for the same joint during the next phases of locomotion, or for the other joints during the same phase of locomotion.

The dual role of natural frequencies in human locomotion can be illustrated by the following example. During the stance period of gait, the ankle joint has to articulate freely for about $1 / 3$ of a second (or with the frequency of $3.33 \mathrm{~Hz}$ ), and then fixed for the same duration [17]. The natural frequency of oscillation in a normal ankle joint is also $3.33 \mathrm{~Hz}$ [18]. Thus, during free articulation, the lower limb is tuned into resonance, and the following fixation of the ankle joint, which is essential for the normal gait pattern, occurs in a resonantly dangerous zone. Stiffness in a joint depends on a balance between a resultant moment generated by the muscles causing rotation and resultant moment from the muscles-antagonists providing resistance to that rotation. There is no sufficient understanding of how the body control system solves this problem constantly. In the meantime we would like to suggest the principle of spectral reciprocity in locomotion as follows.

In locomotion, tuning into resonance to facilitate mobility in one group of joints is reciprocally associated with the tuning out of resonance to facilitate the restriction of mobility in other joints.

\section{IMPLICATION FOR PROSTHETICS AND ORTHOT- ICS}

Criteria (4)-(5) were first applied in the calculating viscoelastic characteristics of a shell, which simulated the effect of orthopedic shoes for patients with cerebral palsy (CP). In a person with a deficiency in coordinating free and fixed mobility in joints, the tuning into-out of resonance requires assistance with technical means. The shoe was represented by a shell with a system of seven specific functional zones with adjustable stiffnesses [19], and a set of exchangeable leaf springs, aimed to tune the "shoe-foot" system out of the unwanted resonance [20].

Another example of the use of the principle of spectral reciprocity was the development of the rolling joint prostheses [21-23], with the stiffnesses in joints being adjustable according to the criteria (4)-(5). A rolling type prosthesis called Free-Flow Foot and Ankle ${ }^{1}$ is shown in Fig. (1). A mechanism for adjustment of stiffness in ankle zone while the tibial component 2 rolls along the foot component $\mathbf{4}$ consists of the pivoted bracket and the side screws $\mathbf{1}$ and elastic buffer 3. Comparative gait studies of 20 below knee amputees demonstrated normalization of gait pattern and increased comfort when a calculated stiffness interval was used in the trials [24].

\section{CONCLUSION}

The principle of spectral reciprocity has been postulated which, we believe, can be instrumental in the development of prosthetics and orthotics. Its application in prosthetic de-

\footnotetext{
${ }^{1}$ Ohio Willow Wood Co., Mt. Sterling Ohio, USA
}

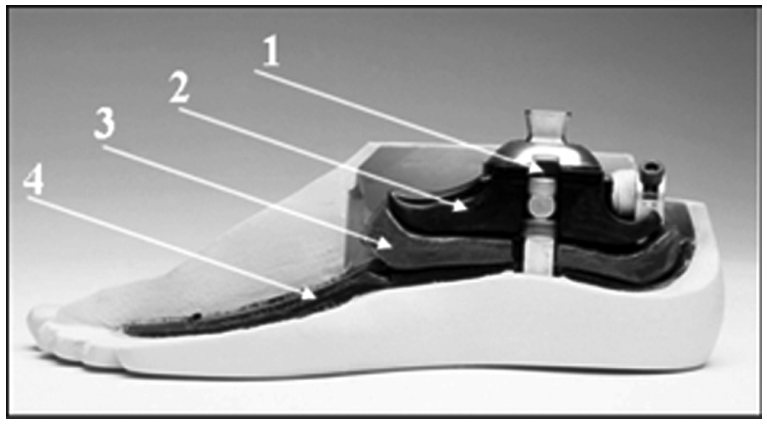

Fig. (1). A rolling type Free-Flow Foot and Ankle: 1 - stiffness control mechanism; 2 - tibial component with the feature of rolling along the foot component $\mathbf{4}$, and the elastic buffer 3 .

sign would better mimic the fundamentals of human biomechanics and thus improve the outcomes of rehabilitation.

\section{ACKNOWLEDGEMENT}

The funding for the study was provided in part by NIH Grant 3R44AR4 3290-03" Prosthetic Rolling Joint Foot and Ankle."

\section{REFERENCES}

[1] Mcmahon, T.A. (1984). Muscles, Reflexes, and Locomotion. Princeton: Princeton University Press.

[2] Lacquaniti, F., Ivanenko, Y.P., Zago, M. (2002). Kinematic control of walking. Arch Ital Biol., 140(4), 263-72.

[3] Stansfield, B.W., Hillman, S.J., Hazlewood, M.E., Robb, J.E. (2006). Regression analysis of gait parameters with speed in normal children walking at self-selected speeds. Gait Posture, 23(3), 288-94.

[4] Aristotle (1934). The Physics. Cambridge, Mass.: Harvard University Press.

[5] Aristotle (1981). On the movement and progression of animals. Studien und materialien zur geschichte der philosophie; Bd. 22. Hildesheim, New York: Olms.

[6] Ottobock. (1999). New Generation Leg System Revolutionizes Lower Limb Prostheses. Orthotics and Prosthetics Business News, 8(19), 47-49.

[7] Desmidt, H.A., Wang, K.W., Smith, E.C. (2002). Coupled TorsionLateral Stability of a Shaft-Disk System Driven Through a Universal Joint. J. Appl. Mech., 69(3), 261-273.

[8] Pitkin, M. (1989). Tuning out of Resonance during Walking and Requirements for Footwear: Proceedings of the XII International Congress of Biomechanics. Los Angeles, CA: 336-7.

[9] Aliev, F.A., Larin, V.B. (1998). Optimization of linear control systems : analytical methods and computational algorithms. Stability and control ; v. 8. Amsterdam, The Netherlands: Gordon and Breach Science Publishers. ix, 261 p.

[10] Abramov, Y.S. (1983). Variational methods in the theory of operator pencils. Leningrad: Leningrad University Press. 178.

[11] Pitkin, M. (1991). New Stride Phases and the Development of Sport Shoe Prototype to Assist the Calf Muscles During Heel-Off: Proceedings of the 15th Annual Meeting, American Society of Biomechanics. Tempe, Arizona: 266-7.

[12] Hof, A.L., Pronk, C.N., Van Best, J.A. (1987). Comparison between EMG to force processing and kinetic analysis for the calf muscle moment in walking and stepping. J Biomech, 20(2), 167-78.

[13] Pitkin, M. (1975). Kinematic and Dynamic Analysis of Human Gait (Rus): Proceedings of the First All-Union Conference in Biomechanics, RNIITO. Riga, Latvia: 279-283.

[14] Duffin, R.J. (1960). The Rayleigh-Ritz method for dissipative or gyroscopic systems. Quart. Appl. Math., 18, 215-221.

[15] Pitkin, M.R. (2006). Biomechanics of lower limb prosthetics (Rus). St. Petersburg, Russia: People and Health, 131 p. Library of Congress Control Number 2006936949.

[16] Vlasov, V.Z., Leontiev, N.N. (1966). Beams, plates and shells on elastic foundations. (Balki, plity i obolochki na uprugom osno- 
vanii). Jerusalem: Israel Program for Scientific Translations; U.S. Clearinghouse for Federal Scientific and Technical Information, Springfield, VA: viii, 357 p. viii, 357 p.

[17] Perry, J. (1992). Gait Analysis: normal and pathological function. Thorofare, NJ: Slack, Inc.

[18] Mccronville, J., Churchill, T., Kaleps, I., Clauser, C., Cuzzi, J. (1980). Anthropometric relationships of body and body segments moments of inrtia. (Report No. AFAMRL-TR-80-119). Yellow Springs, OH: Anthropology Research Project, Inc.

[19] Pitkin, M. (1986). Biomechanical Requirements for Polymer Orthopedic Shoes (Rus). Prostheses and Prosthetics, 76, 83-91.

[20] Mendelevich, I., Pitkin, M. (1989). Orthopedic shoes for children with cerebral palsy in residual stage and with "pes equino-varus congenital" which is feebly marked or was surgically treated: Pro- ceedings of the XII International Congress of Biomechanics. Los Angeles, CA: 375-6.

[21] Pitkin, M. (1995). Artificial Knee Having Dual Flexion Action During Locomotion. U.S. Patent No. 5,405,408. Washington, D.C.: U.S. Patent and Trademark Office.

[22] Pitkin, M., Hays, J., Srinivasan, S., Colvin, J. (2001). Artificial foot and ankle U.S. Patent No. 6,290,730. Washington, D.C.: U.S. Patent and Trademark Office.

[23] Pitkin, M. (1995). Mechanical outcome of a rolling joint prosthetic foot, and its performance in dorsiflexion phase of the trans-tibial amputee gait. J. Prosthet. Orthot., 7(4), 114-123.

[24] Pitkin, M., Colvin, J., Hayes, J. (1999). Gait analysis of twenty unilateral transtibial amputees. (Report NIH/NIAMS/NCMRR Grant 3R44AR4 3290-03). Mt. Sterling, OH: Ohio Willow Wood Co. 